\title{
The Chitranjan Ranawat Award
}

\section{Should Prophylactic Antibiotics Be Withheld Before Revision Surgery to Obtain Appropriate Cultures?}

\author{
Matthew W. Tetreault BA, Nathan G. Wetters MD, Vinay Aggarwal BS, \\ Michael Mont MD, Javad Parvizi MD, FRCS, Craig J. Della Valle MD
}

Published online: 30 April 2013

(C) The Association of Bone and Joint Surgeons ( 2013

\begin{abstract}
Background Preoperative antibiotics are known to be critical for decreasing the risk of periprosthetic joint infection (PJI) in primary THA and TKA. However, antibiotics often are withheld before revision surgery, as there is concern that even a single dose of prophylactic antibiotics may affect intraoperative cultures.

Questions/purposes In this prospective randomized controlled trial, we determined the effect of a single dose of
\end{abstract}

prophylactic antibiotics on cultures obtained at the time of revision arthroplasty.

Methods We randomized 65 patients with known PJI after 37 TKAs and 28 THAs at three centers. Patients were included in the trial if they had a culture-positive aspiration and had not taken antibiotics within 2 weeks of the procedure. Patients were randomized to receive prophylactic antibiotics either before the skin incision or after a minimum of three sets of intraoperative cultures were obtained. Preoperative and

The institution of one or more of the authors has received, during the study period, funding from Zimmer Inc (Warsaw, IN, USA) (MWT, NGW, CDV), and Orthopaedic Research and Education Foundation (Rosemont, IL, USA) (MM).

One of the authors certifies that he (MM) has received or may receive payments, during the study period, an amount of USD 100,000 to $100,000,000$ from Stryker Orthopaedics (Mahwah, NJ, USA), an amount of less than USD 10,000 from Wright Medical Technology, Inc (Arlington, TN, USA), an amount of less than USD 10,000 from Biocomposites Inc (Wilmington, NC, USA), an amount of USD 10,000 to 100,000 from Jannsen, Inc (Toronto, ON, CA), an amount of less than USD 10,000 from Joint Active Systems, Inc (Effingham, IL, USA), an amount of less than USD 10,000 from Medtronic, Inc (Minneapolis, MN), an amount of USD 10,000 to 100,000 from Sage Products LLC (Cary, IL, USA), an amount of USD 10,000 to 100,000 from DJ Orthopedics (Vista, CA, USA), and an amount of USD 10,000 to 100,000 from TissueGene (Rockville, MD, USA).

One of the authors certifies that he (JP) has received or may receive payments or benefits, during the study period, an amount of USD 10,000 to 100,000 from Smith \& Nephew, Inc (Memphis, TN, USA), an amount of less than USD 10,000 from 3 M (St. Paul, MN, USA), an amount of less than USD 10,000 from TissueGene (Rockville, MD, USA), an amount of USD 10,000 to USD 100,000 from Zimmer Inc (Warsaw, IN, USA), an amount of USD 10,000 to USD 100,000 from Convatec (Skillman, NJ, USA), an amount of USD 10,000 to USD 100,000 from CeramTec (Laurens, SC, USA), an amount of less than USD 10,000 from Emovi Inc (Laval, Quebec, CA), an amount of less than USD 10,000 from Cadence Pharmaceuticals, Inc. (San

Diego, CA, USA), an amount of less than USD 10,000 from Medtronic, Inc (Minneapolis, MN), and an amount of less than USD 10,000 from Pfizer (New York, NY, USA).

One of the authors certifies he (CDV) has received or may receive payments or benefits, during the study period, an amount of USD 10,000 to 100,000 from Biomet Inc (Warsaw, IN, USA), an amount of USD 10,000 to 100,000 from Smith \& Nephew, Inc (Memphis, TN, USA), an amount of USD 10,000 to 100,000 from Stryker Orthopaedics (Mahwah, NJ, USA), and an amount of USD 10,000 to 100,000 from CD Diagnostics (Wynnewood, PA, USA). All ICMJE Conflict of Interest Forms for authors and Clinical Orthopaedics and Related Research editors and board members are on file with the publication and can be viewed on request. Clinical Orthopaedics and Related Research neither advocates nor endorses the use of any treatment, drug, or device. Readers are encouraged to always seek additional information, including FDA approval status, of any drug or device before clinical use.

Each author certifies that his or her institution approved the human protocol for this investigation, that all investigations were conducted in conformity with ethical principles of research, and that informed consent for participation in the study was obtained.

This work was performed at Rush University Medical Center (Chicago, IL, USA), Rothman Institute of Orthopedics (Philadelphia, PA, USA), and Sinai Hospital of Baltimore (Baltimore, MD, USA).

Electronic supplementary material The online version of this article (doi:10.1007/s11999-013-3016-5) contains supplementary material, which is available to authorized users. 
intraoperative cultures were then compared. Results between patients who did and did not receive antibiotics were compared using an equivalence test for proportion differences (two one-sided t-tests [TOST]) with a 0.2 margin.

Results Intraoperative cultures yielded the same organisms as preoperative cultures in 28 of 34 patients (82\%) randomized to receive antibiotics before the skin incision compared to 25 of 31 patients $(81 \%)$ randomized to receive antibiotics after obtaining operative cultures (statistically equivalent by TOST estimate: $\mathrm{p}=0.0290$ ).

Conclusions In this randomized controlled trial, there was no effect on the results of cultures obtained intraoperatively when prophylactic antibiotics were administered before skin incision. Given the known benefits of prophylactic antibiotics in preventing PJI, preoperative prophylaxis should not be withheld in revision surgery for fear of affecting cultures. Level of Evidence Level I, therapeutic study. See Instructions for Authors for a complete description of levels of evidence.

\section{Introduction}

The administration of preoperative, prophylactic antibiotics is the standard of care for primary THA and TKA, having been shown to significantly reduce the risk of periprosthetic joint infections (PJIs) [4, 5, 7]. Nevertheless, preoperative antibiotics are sometimes withheld in patients undergoing revision arthroplasty, as there is concern that occult infection may be present and the administration of antibiotics might affect intraoperative culture results [7, 10]. This would be important if true, as culture results are integral to the diagnosis of PJI and antibiotic sensitivities obtained from these cultures are critical for guiding subsequent antimicrobial therapy.

A recent paper by Burnett et al. [2] suggested preoperative antibiotics should not be withheld before revision TKA surgery because culture results were not affected by a single dose of prophylactic antibiotics. Their prospective,

M. W. Tetreault, N. G. Wetters, C. J. Della Valle Department of Orthopaedic Surgery, Rush University Medical Center, Chicago, IL, USA

C. J. Della Valle $(\bowtie)$

Midwest Orthopaedics at Rush, 1611 W Harrison Street,

Suite 300, Chicago, IL, USA

e-mail:craigdv@yahoo.com

V. Aggarwal, J. Parvizi

Rothman Institute of Orthopedics, Philadelphia, PA, USA

M. Mont

Department of Orthopaedic Surgery, Sinai Hospital

of Baltimore, Baltimore, MD, USA nonrandomized study enrolled 25 patients with 26 infected TKAs with a known preoperative infecting organism and no recent antibiotic therapy. All patients had repeat aspiration of the infected TKA performed in the operating room before prophylactic antibiotic administration and then tissue cultures after antibiotics at the time of open arthrotomy. A $100 \%$ concordance rate was reported between preantibiotic and postantibiotic cultures.

We designed the current study to build on that important work by including both hip and knee arthroplasties, increasing sample size, and randomizing patients. Specifically, we determined whether there was a difference in the concordance rate between preoperative and intraoperative cultures between patients with known PJIs who were randomized to receive a single dose of antibiotics either before skin incision or after obtaining intraoperative cultures.

\section{Patients and Methods}

After obtaining institutional review board approval, this study was registered as a randomized controlled trial (clinicaltrials.gov study identifier: NCT01174212). We prospectively screened 102 consecutive patients with clinical evidence of PJI as defined by the criteria of Parvizi et al. [9] at three sites between January 2010 and April 2012. Thirty-seven patients were excluded, including 29 with clinical evidence of infection but negative preoperative cultures upon aspiration, five who had received antibiotic therapy within 2 weeks of preoperative aspiration, two with draining sinuses but inadequate aspirates for preoperative cultures, and one with septic arthritis of a native knee, leaving 65 patients for randomization. Of these 65 patients, 24 were women (37\%) and 41 were men (63\%). The mean age for the cohort was 61 years (range, 43-92 years). Twenty-eight patients underwent revision THA (43\%) and 37 underwent revision TKA (57\%). Thirteen patients were being treated for acute postoperative infections ( $\leq 6$ weeks postoperatively; 20\%), 32 were chronic infections (49\%), and 20 were acute hematogenous infections (acute onset of pain in a previously well-functioning joint arthroplasty associated with fever and/or an obvious site of infection such as a pneumonia or a cutaneous abscess; $31 \%)$. Fifteen patients $(23 \%)$ were treated with débridement and exchange of liner or modular components, 48 (74\%) had placement of an antibiotic spacer, and two patients $(3 \%)$ had a one-stage revision of components. Original diagnoses for the primary procedure included osteoarthritis (60), femoral neck fracture (two), rheumatoid arthritis (one), osteonecrosis (one), and osteomyelitis (one).

After signing informed consent and before surgical intervention for the treatment of PJI, patients were 
randomized to receive prophylactic intravenous antibiotics ( $2 \mathrm{~g}$ cefazolin and $1 \mathrm{~g}$ vancomycin intravenously) either (1) within 1 hour before incision or (2) after obtaining intraoperative cultures. Sequentially numbered randomization cards were developed by a statistician who had performed a power analysis and opened once patients were anesthetized. In the group receiving antibiotics before cultures, antibiotics were always infused before application and inflation of the pneumatic tourniquet for revision TKAs. Thirty-four patients were given antibiotics before cultures were obtained and 31 received prophylactic antibiotics after intraoperative cultures. The composition of the two groups was statistically similar by age, sex distribution, revision TKA versus THA percentage, infection type (chronic versus acute), and procedure type (Table 1).

At least three cultures were collected from within the joint during surgery (including multiple swab and tissue cultures), and culture results in both study populations were compared with preoperative cultures. Culture results were recorded once they were declared final by the microbiology laboratory of each respective institution. A positive preoperative culture was considered growth from solid medium; growth from broth only was excluded. There were no instances where a broth-only culture was duplicated by other cultures from the same patient. All culture results were compared, including both organisms and their sensitivities. In addition, a microbiologist with expertise in bone and joint infection was consulted to clarify probable culture contaminants.

Demographic differences were evaluated using Tukey t-tests and chi-square tests of independence. Risk estimates were calculated and reported with $95 \%$ exact confidence intervals. A power analysis was performed with an expected power of 0.80 and an alpha of 0.05 sensitive to

Table 1. Baseline characteristics by randomization group

\begin{tabular}{llll}
\hline $\begin{array}{l}\text { Patient-specific } \\
\text { variable }\end{array}$ & $\begin{array}{l}\text { Antibiotics given } \\
\text { preoperatively } \\
(\mathrm{n}=34)\end{array}$ & $\begin{array}{l}\text { Antibiotics held for } \\
\text { intraoperative culture } \\
(\mathrm{n}=31)\end{array}$ & P value \\
\hline $\begin{array}{l}\text { Age (years)* } \\
\begin{array}{l}\text { Male (number of } \\
\text { patients) }\end{array}\end{array}$ & $20.5 \pm 10.5$ & $63.1 \pm 8.9$ & 0.1433 \\
$\begin{array}{c}\text { TKA (number of } \\
\text { patients) }\end{array}$ & $19(55.9 \%)$ & $18(58.1 \%)$ & 0.4568 \\
$\begin{array}{c}\text { Chronic } \\
\text { infections } \\
\text { (number of } \\
\text { patients) }\end{array}$ & $16(47.1 \%)$ & $16(51.6 \%)$ & 0.8592 \\
$\begin{array}{c}\text { Components } \\
\text { retained } \\
\text { (number of } \\
\text { patients) }\end{array}$ & $9(26.5 \%)$ & $6(19.4 \%)$ & 0.7138 \\
\hline
\end{tabular}

* Values are expressed as mean $\pm \mathrm{SD}$. proportional difference of $15 \%$ between groups. Our allowable concordance range was $80 \%$ to $100 \%$ using reported values $[1-3,8]$, resulting in a sample size requirement of 27 patients per randomization group (54 patients total). Equivalence of risk estimates was tested using two one-sided tests (TOST) with a margin of 0.2 . The TOST method effectively tests the hypothesis that two estimates are equivalent within a statistically computed equivalence range. The lower the $\mathrm{p}$ value is, the more similar the estimates can be concluded to be, and if the $p$ value is less than the critical cutoff $(0.05)$, then the two estimates can be assumed to be statistically equivalent. Statistical tests with $\mathrm{p}$ values of less than 0.05 were considered significant.

\section{Results}

The use of preoperative antibiotics did not appear to affect culture results. Of the patients receiving preoperative antibiotics (Appendix 1; supplemental materials are available with the online version of $\left.\mathrm{CORR}^{\circledR}\right), 28$ of $34(82 \%$; 95\% CI: $65.5 \%-93.2 \%$ ) had identical preoperative and intraoperative culture results while 25 of 31 (81\%; $95 \% \mathrm{CI}$ : $62.5 \%-92.6 \%$ ) patients with antibiotics held (Appendix 2; supplemental materials are available with the online version of $\mathrm{CORR}^{\circledR}$ ) showed identical cultures (TOST $\mathrm{p}=0.0290)$. The culture concordance rate was slightly lower for hips $(78.6 \%$; 95\% CI: $59.1 \%-91.7 \%)$ than for knees $(83.8 \%$; 95\% CI: $68.0 \%-93.8 \%$ ); this difference was neither statistically significantly different $(\mathrm{p}=0.5917)$ nor significantly equivalent. With the numbers available, the threshold of equivalence was not met on the TOST equivalence test, although it was close (TOST $\mathrm{p}=0.0665)$.

\section{Discussion}

The use of preoperative antibiotic prophylaxis before surgery has been shown to reduce the incidence of postoperative infection and is the standard of care for primary THA and TKA [4, 5, 7]. However, many surgeons remain concerned that, for revision procedures, preoperative antibiotics may alter intraoperative culture results, which can make it more difficult to either diagnose a PJI or target antibiotic therapy [7, 10]. In our study, we determined whether there was a difference in the concordance rate between preoperative and intraoperative cultures between patients with known PJIs who were randomized to receive antibiotics either before skin incision or after obtaining intraoperative cultures.

There were several limitations of this study. Although our study was powered at $80 \%$, there is still a $20 \%$ chance of not 
identifying a true effect; with a larger sample size, we would be more likely to identify true equality in concordance rates. However, our sample is larger than that reported by Burnett et al. [2] and our data suggest, even if there is a difference between the two strategies, it is small and potentially not clinically important enough to withhold prophylactic antibiotics given the known benefits of administration before the incision. In addition, we studied a specific population (those with a known PJI) and are extrapolating our results to all patients undergoing revision procedures. Further studies could include randomization of prophylactic antibiotics to a more general population of patients undergoing revision for any reason (including infection) to compare culture concordance and risk of subsequent PJI, although this would require a much larger sample size and logistically would be more difficult to complete. Similarly, our relatively small patient population did not allow us to examine the effect of antibiotic administration on different organisms; that is, certain organisms may be more or less sensitive to even one single dose of prophylactic antibiotics, which is another area for further study. Finally, we excluded cultures that were positive for broth only, which could have changed our results; however, in our data set, the inclusion of these results would not have changed our concordance rates. We chose not to include these results based on the Musculoskeletal Infection Society definition of PJI.

We found no difference in the concordance rate between preoperative and intraoperative cultures between patients with known PJIs who were randomized to receive antibiotics either before skin incision or after obtaining intraoperative cultures. Based on these findings, we believe prophylactic antibiotics should be given routinely before revision procedures. Previous work has shown the risk of subsequent PJI is higher after revision than after primary procedures $[6,11]$. While this risk discrepancy is likely multifactorial, withholding prophylactic antibiotics until after cultures are obtained intraoperatively may contribute to the higher rates of PJI observed in the revision population. Given the clear benefit of prophylactic antibiotic administration before skin incision and our findings, which are in concordance with those of Burnett et al. [2], we believe the benefits of prophylaxis outweigh the potential risks of affecting culture results; therefore, prophylactic antibiotics should be given in the majority of cases.

Overall, we found a lower rate of agreement between preoperative and intraoperative cultures compared to prior studies. A 2007 retrospective analysis of 171 infected TKAs reported a false-negative rate intraoperatively of $12.5 \%$ in patients who received antibiotics compared to $8 \%$ in those with antibiotics withheld [3]. Additional organisms were cultured intraoperatively in $4 \%$ versus $1.4 \%$ of patients in the two groups. Both differences were statistically insignificant. A more recent prospective study by the same senior author reported a $100 \%$ concordance rate with infusion of antibiotics preoperatively (26 infected TKAs total) [2]. Our concordance rates of $82.4 \%$ and $80.6 \%$ in the antibiotics before and after groups, respectively, while lower than published values, were statistically equivalent between groups. One reason for our higher rate of disagreement may be related to prior work not comparing organism antibiotic sensitivity profiles; specifically, we found Staphylococcus epidermidis was the most common organism to change sensitivities from preoperative to intraoperative cultures (eg, methicillin-sensitive to methicillin-resistant; methicillin-resistant to methicillinsensitive, or methicillin-resistant to both methicillin-sensitive and methicillin-resistant). Thus, cultures classified as discordant may have been classified as concordant in the cited studies. Our sample included hips in addition to knees and the increased technical difficulty associated with hip over knee aspiration could have contributed to the lower concordance rates.

In conclusion, our data support prior reports that a single dose of prophylactic antibiotics does not alter intraoperative culture results in PJI. Thus, we do not recommend prophylactic antibiotics be withheld before revision hip and knee arthroplasty given their known benefit in the prevention of PJI.

Acknowledgments The authors thank Dr John Segreti for lending his microbiology expertise, Dr Robert Pivec for aiding in data collection, and Mario Moric MS for his help with statistical analysis.

\section{References}

1. Ali F, Wilkinson JM, Cooper JR, Kerry RM, Hamer AJ, Norman $\mathrm{P}$, Stockley I. Accuracy of joint aspiration for the preoperative diagnosis of infection in total hip arthroplasty. J Arthroplasty. 2006;21:221-226.

2. Burnett RS, Aggarwal A, Givens SA, McClure JT, Morgan PM, Barrack RL. Prophylactic antibiotics do not affect cultures in the treatment of an infected TKA. Clin Orthop Relat Res. 2010; 468:127-134.

3. Ghanem E, Parvizi J, Clohisy J, Burnett S, Sharkey PF, Barrack R. Perioperative antibiotics should not be witheld in proven cases of periprosthetic infection. Clin Orthop Relat Res. 2007; 461:44-47.

4. Hanssen AD, Osmon DR. The use of prophylactic antimicrobial agents during and after hip arthroplasty. Clin Orthop Relat Res. 1999;369:124-138.

5. Hanssen AD, Osmon DR, Nelson CL. Prevention of deep periprosthetic joint infection. Instr Course Lect. 1999;48:111-122.

6. Hanssen AD, Rand JA. Evaluation and treatment of infection at the site of a total hip or knee arthroplasty. Instr Course Lect. 1999;48:111-122.

7. Mangram AJ, Horan TC, Pearson ML, Silver LC, Jarvis WR. Guideline for prevention of surgical site infection, 1999. Centers for Disease Control and Prevention (CDC) Hospital Infection Control Practices Advisory Committee. Am J Infect Control. 1999;27:97-132; quiz 133-134; discussion 96. 
8. Müller M, Morawietz L, Hasart O, Strube P, Perka C, Tohtz S. Diagnosis of periprosthetic infection following total hip arthroplasty - evaluation of the diagnostic values of pre- and intraoperative parameters and the associated strategy to preoperatively select patients with a high probability of joint infection. J Orthop Surg Res. 2008;3:31.

9. Parvizi J, Zmistowski B, Berbari EF, Bauer TW, Springer BD, Della Valle CJ, Garvin KL, Mont MA, Wongworawat MD, Zalavras CG. New definition for periprosthetic joint infection: from the Workgroup of the Musculoskeletal Infection Society. Clin Orthop Relat Res. 2011;469:2992-2994.

10. Spangehl MJ, Masri BA, O'Connell JX, Duncan CP. Prospective analysis of preoperative and intraoperative investigations for the diagnosis of infection at the sites of two hundred and two revision total hip arthroplasties. J Bone Joint Surg Am. 1999;81:672-683.

11. Winiarsky $\mathrm{R}$, Barth $\mathrm{P}$, Lotke P. Total sknee arthroplasty in morbidly obese patients. J Bone Joint Surg Am. 1998;80: 1770-1774. 